\title{
Decreasing Incidence of Alcohol-Related Liver Disease in Denmark: A 25-Year Nationwide Study
}

This article was published in the following Dove Press journal:

Clinical Epidemiology

\author{
Frederik Kraglund (D) \\ Thomas Deleuran ${ }^{1,2}$ \\ Gro Askgaard ${ }^{1,3}$ \\ Kate M Fleming (D) ${ }^{4,5}$ \\ Peter Jepsen $\mathbb{D}^{1,6}$
}

'Department of Hepatology and Gastroenterology, Aarhus University Hospital, Aarhus, Denmark; ${ }^{2}$ Department of Medicine, Randers Regional Hospital, Randers, Denmark; ${ }^{3}$ Department of Internal Medicine, Zealand University Hospital, Køge, Denmark; ${ }^{4}$ Institute of Population Health, University of Liverpool, Liverpool, UK; ${ }^{5}$ Liverpool Centre for Alcohol Research, University of Liverpool, Liverpool, UK;

${ }^{6}$ Department of Clinical Epidemiology, Aarhus University Hospital, Aarhus, Denmark
Correspondence: Frederik Kraglund Palle Juul-Jensens Boulevard 99, Aarhus 8200

Email frekra@clin.au.dk
Purpose: Alcohol consumption has decreased in Denmark in recent years. We aimed to illustrate and investigate the developments in the incidence, hospital care, and mortality of alcohol-related liver disease (ALD) in Denmark during the last 25 years.

Patients and Methods: Through nationwide healthcare registries, we identified all Danish patients with incident ALD in 1994-2018. We computed standardized incidence rates by sex, age, and geography, age-specific incidence rates by birth cohort, and standardized prevalence. We enumerated inpatient admissions, days of admission, outpatient visits, and emergency room visits. Lastly, we estimated relative risks of mortality, standardized mortality rates, and the proportion of deaths caused by ALD.

Results: The standardized incidence rate decreased from its peak at 357 per 1,000,000 in 2009 to 240 per 1,000,000 in 2018, and the decrease was evident for both sexes and all age groups below 70 years. The standardized prevalence was stable around $0.22 \%$ from 2011 onwards. There was an almost fivefold geographic variation in standardized incidence by municipalities, and age-specific incidence rates decreased sequentially with each 5-year birth cohort after 1960 . The number of inpatient admissions, days of admission, and emergency room visits decreased during the study period, while the number of outpatient visits was stable. For patients diagnosed in 2014-2018 compared to 1994-1998, the relative risk of 1-year mortality was 0.83 (95\% confidence interval: $0.78-0.87$ ), and the standardized mortality along with the proportion of deaths caused by ALD decreased during the study period.

Conclusion: The incidence of ALD decreased from 357 to 240 per 1,000,000 over the last 10 years in Denmark. During the same period, the prevalence remained stable around $0.22 \%$ and mortality decreased. Additionally, the burden of ALD on hospital care decreased significantly between 1994 and 2018. We anticipate a further decrease in the incidence of ALD in the future.

Keywords: alcohol-related liver disease, epidemiology, time trends, public health, incidence, healthcare, mortality, cause of death

\section{Plain Language Summary}

We studied the epidemiology of alcohol-related liver disease in Denmark from 1994 to 2018. During this 25-year period, the annual number of Danes diagnosed with alcohol-related liver disease has decreased. Meanwhile, the prognosis for Danish patients with alcohol-related liver disease has improved: patients have fewer hospital admissions and live longer.

\section{Introduction}

Alcohol-related liver disease (ALD) encompasses any liver injury caused by alcohol, from simple steatosis to cirrhosis. ${ }^{1}$ Though alcohol-attributable mortality has come down in Denmark in recent years, it remains high compared to other Nordic 
countries. ${ }^{2}$ The global burden of ALD is substantial, ${ }^{3}$ and in Denmark in 2017, 10\% of disability-adjusted life-years (DALYs) lost were attributable to alcohol use. ${ }^{4}$ The relationship between alcohol consumption and liver injury is dose-dependent, ${ }^{5}$ but factors such as daily drinking, drinking outside meals, and obesity also affect individual-level risk. ${ }^{6-8}$ At the population level, the incidence of ALD is closely linked with alcohol consumption. ${ }^{9}$ Therefore, governments may reduce the burden of ALD by increasing alcohol taxation and reducing availability, and by implementing screening and brief interventions for harmful alcohol use in primary care. ${ }^{9,10}$

We have previously studied the epidemiology of ALD in Denmark in $1988-2013 .{ }^{11,12}$ In those studies, we saw a stable incidence, but predicted a future decrease in the incidence of ALD based on birth cohort analyses. ${ }^{11,12}$ Moreover, per capita alcohol consumption has come down since our last study. ${ }^{13}$

With this registry-based study, we aimed to illustrate and investigate the developments in the incidence, hospital care, and mortality of ALD in Denmark from 1994 through 2018. Such measures are valuable in guiding alcohol prevention strategies and monitoring public health.

\section{Patients and Methods}

\section{Setting}

This nationwide, registry-based cohort study was conducted in the Danish population of 5,781,190 people (1 January 2018). In Denmark, citizens are provided taxsupported, universal health care, which grants equal access to general practitioners and hospitals. All Danish citizens are issued a personal identification number at birth or immigration, which allows linkage across all national registries. The Danish National Patient Registry was established in 1977. It contains data from all inpatient hospitalizations including primary and secondary discharge diagnosis codes as well as procedure codes going back to 1977 . Outpatient visits are registered from 1994 onwards, and emergency room visits are registered from 1995 onwards. ${ }^{14,15}$ The Danish Civil Registration System records vital status with complete follow-up from birth or immigration until death or emigration, ${ }^{14}$ and the Danish Register of Causes of Death records immediate, supplementary, and underlying causes of death. ${ }^{16}$

\section{Study Population}

We identified all Danish patients with a first diagnosis of ALD from 1977 to 2018 in the Danish National Patient
Registry using the diagnosis codes for cirrhosis due to alcohol-related liver disease (ALD cirrhosis) (ICD-8: 571.09, ICD-10: K70.2, K70.3, and K70.4), alcoholic hepatitis (ICD-10: K70.1), steatosis due to alcoholrelated liver disease (ALD steatosis) (ICD-8: 571.10, ICD-10: K70.0), and unspecified ALD (ICD-10: K70.9). If, at that time, the patient received diagnosis codes for more than one of these conditions, we used the following hierarchy: cirrhosis, hepatitis, steatosis, unspecified. We limited the study period to 1994-2018 (the ICD-10 era) to ensure homogeneity of coding practice. ${ }^{15}$ We used data from 1977-1993 to avoid misclassifying patients with prevalent ALD as patients with incident ALD, and we used data from 2019 on vital status to add an additional calendar year of follow-up. In the analysis of emergency room visits, the study period was limited to 1995-2018. We defined ALD-related mortality as either of the following underlying causes registered in the Register of Causes of Death: liver cirrhosis (ICD-10: K74.x), ALD (ICD-10: K70.x), alcohol misuse (ICD-10: F10.x), and hepatocellular carcinoma (ICD-10: C22.x).

\section{Statistical Analysis}

For each year, we computed the incidence rate as the number of patients with a first-time ALD diagnosis during the year divided by the number of Danish citizens at the beginning of the same year. We used direct standardization to adjust the annual incidence rates for changes in the sexand age group-distribution of the Danish population using the Danish population on 1 January 2018 as the standard (Supplementary Table S1). In this manner, we computed overall and stratum-specific standardized incidence rates by sex and age group $(<40,40-49,50-59,60-69, \geq 70)$. To analyse the geographic distribution of ALD, we computed mean annual incidence rates of ALD for each of the 98 Danish municipalities during the last 10 years (2009-2018). We then standardized these rates to the sex- and age group-distribution of the entire Danish population on 1 January 2018 using direct standardization (Supplementary Table S1). Moreover, to compare the agespecific incidence rates across birth cohorts, we computed incidence rates by 5 -year birth cohorts born between 1930 and 1979 and 5-year age groups from 30 to 79 years. We used age to estimate the distribution of birth years. For instance, the number of people born in 1960-1964 and alive at age 40-44 was approximated as the number of people aged 40-44 in year 2000-2004. 
We computed overall and sex-specific prevalence by dividing the number of patients with ALD on 1 January of a given year with the Danish population on the same day. We then standardized to the sex- and age-distribution of the Danish population on 1 January 2018 using direct standardization.

The number of inpatient admissions per patient with ALD each year was estimated as the number of all-cause inpatient admissions (excluding transfers between hospital departments) divided by the person-years at risk in a given calendar year. Similarly, we estimated the number of ALD-related outpatient visits and all-cause emergency room visits per person-year at risk. ALD-related outpatient visits were defined as any of the following: outpatient visits with diagnosis codes for ALD (including alcoholrelated hepatic encephalopathy) (ICD-10: K70, ICD-8: 571.09 or 571.10), ascites (ICD-10: R18.x), or esophageal or gastric varices (ICD-10: I85.x and I86.4) or procedure codes for banding of or sclerotherapy for esophageal varices (NCSP: JCA20, JCA22 and JCA32) or diagnostic or therapeutic paracentesis (NCSP: TJA10.x) in patients with ALD. For every year, we also estimated the total number of days spent in hospital as an inpatient per person-year and the average length of admission. The route of diagnosis was defined as either emergent (during emergency room visit or at emergent inpatient admission) or elective (during outpatient visit or at elective admission) dependent on where the patient received their first primary diagnosis code for ALD.

We do not report confidence intervals in the analyses of incidence, prevalence, and hospital care since these analyses are enumerations of complete data and not statistical estimates. Hence, there is no statistical uncertainty to account for in these analyses. ${ }^{17}$

We used the Kaplan-Meier method to estimate crude all-cause mortality with respect to time since first ALD diagnosis by sex, age group, ALD diagnosis, and calendar time period. We further estimated relative risks (RR) of 1and 5-year all-cause mortality by sex (female [reference], male), age group ( $<40$ years [reference], 40-59 years, 60-69 years, $>70$ years), ALD diagnosis (steatosis [reference], unspecified, hepatitis, cirrhosis), and calendar time period (1994-1998 [reference], 1999-2003, 2004-2008, 2009-2013, 2014-2018) by generating pseudoobservations to fit a generalized linear model. ${ }^{18}$ Within each stratum we adjusted the RRs for confounding by the other covariates, so the effect of one covariate (eg sex) on all-cause mortality was adjusted for differences in the other three covariates (eg age group, ALD diagnosis, and calendar time period). To increase the comparability of calendar time periods, we made their lengths of follow-up equal, so for instance, patients diagnosed with ALD in 1994-1998 were censored on 31 December 1999, and patients diagnosed in 2014-2018 were censored on 31 December 2019. To investigate time trends in mortality further, we estimated 1-, 3-, and 5-year mortality risk by calendar year, sex, age group, and ALD diagnosis using the Kaplan-Meier method. We then adjusted for changes in the population of patients with ALD over calendar time using direct standardization. For each year, we standardized the expected number of deaths in each stratum to the distribution of sexes, age groups, and ALD diagnoses of patients diagnosed in 2013 (Supplementary Table S2). We repeated the analysis of standardized mortality, stratified by the route of diagnosis. For each year, we calculated the proportion of 1-year mortality that could be attributed to ALD among patients with established ALD. This measure was chosen to avoid bias by differing follow-up times among patients with ALD.

Lastly, to test the extent to which diagnoses of ALD steatosis affected the results, we repeated all aforementioned analyses excluding all diagnoses of ALD steatosis.

\section{External Data}

To provide context for our findings, we included survey data from 2010, 2013, and 2017 on high-risk alcohol consumption from the Danish National Health Surveys. ${ }^{19}$ High-risk alcohol consumption is defined as $>252 / 168$ grams per week for men/women by the Danish Health Authority. Additionally, we used data collected by Statistics Denmark on the yearly sales of pure alcohol during the study period. These data cover all domestic taxed sales and cross-border trade with alcohol. ${ }^{13}$

\section{Results}

We identified 41,316 Danish patients with a first-time diagnosis of ALD between 1994 and 2018. During this period, the median age at first diagnosis increased from 52 (interquartile range: 45-61) to 62 years (interquartile range: 55-70), and the proportion of men was stable around $68 \%$. Overall, $69 \%$ had ALD cirrhosis at the time of the first ALD diagnosis, $14 \%$ had ALD steatosis, $10 \%$ had unspecified ALD, and 7\% had alcoholic hepatitis. The distribution of diagnoses did not change throughout the study period (Supplementary Figure S1). During 219,796 person-years of follow-up, 30,393 
patients died, and the proportion of deaths caused by ALD decreased from $76 \%$ in 1994 to $60 \%$ in 2018 .

\section{Incidence and Prevalence}

The overall standardized incidence rate (SIR) of ALD peaked at 357 per $1,000,000$ in 2009. Since then, it decreased steadily to 240 per $1,000,000$ in 2018 , corresponding to a ratio of 0.67 (Figure 1). Each year, the SIR was about twice as high for men as for women. For women, the SIR decreased from 225 in 2009 to 146 in 2018 (ratio: 0.65), and for men, it decreased from 491 in 2009 to 335 in 2018 (ratio: 0.68) (Figure 1). We observed a decrease in ALD incidence in both sexes and all age groups below 70 years, and the decrease was most pronounced in women aged 40-49 (ratio: 0.32, difference: -176 per $1,000,000$ ), women aged 50-59 (ratio: 0.43, difference: -363 per $1,000,000$ ), and in men aged $40-49$ (ratio: 0.51 , difference: -176 per $1,000,000$ ). There was a slight increase in the ALD incidence of women aged 70 or more (ratio: 1.06, difference: 15 per 1,000,000) (Figure 2). The overall standardized prevalence of ALD was stable around $0.22 \%$ since 2011. In 2018, the prevalence of ALD was $0.29 \%$ in men and $0.16 \%$ in women.

We observed large geographic variation between municipalities. The mean annual SIRs peaked in small island communities where we observed the highest rates of 669 , 638 , and 570 per $1,000,000$. We observed the lowest rates of 144,150 , and 155 per 1,000,000 in rural areas in the middle of Jutland. Overall, the eastern part of Denmark had higher rates than the western part (Figure 3).

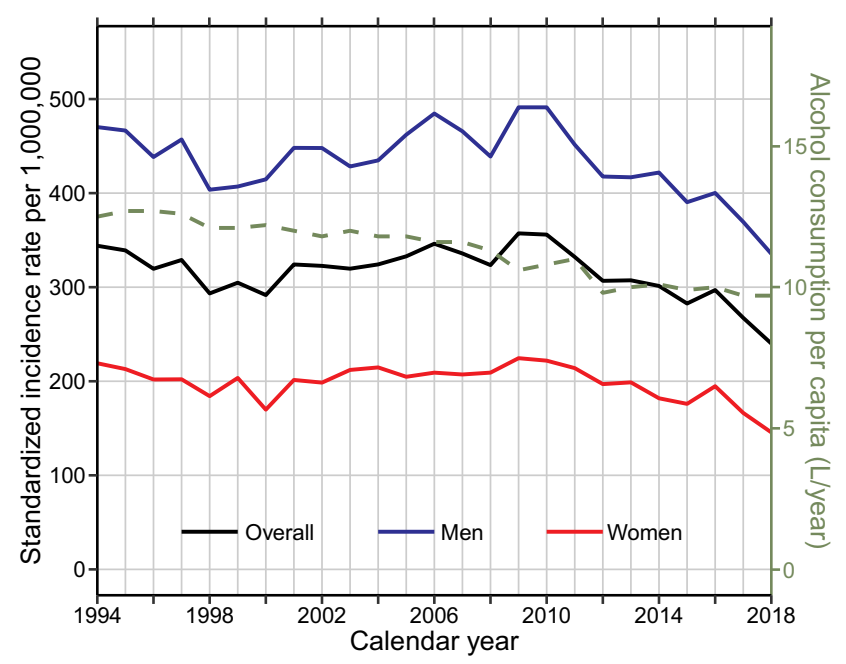

Figure I Time trends in annual standardized incidence rates per I,000,000 Danish population 1994-2018 (left $y$-axis) and annual alcohol sales per Danish citizen $>18$ years in liters of pure alcohol 1994-2018 (right y-axis).
Regarding 5-year birth cohorts, the 1950-1959 birth cohorts had the highest age-specific incidence rates, and the age-specific incidence rates decreased sequentially with each following birth cohort (Figure 4 and Supplementary Figure S2).

\section{Hospital Care}

The number of all-cause inpatient admissions for patients with ALD per person-year decreased from 2.05 in 1994 to 1.54 in 2000, was stable until 2010, and then decreased further to 1.12 in 2018 (Figure 5, top left). The total number of inpatient days for patients with ALD per person-year decreased from 17.3 in 1994 to 7.1 in 2018 (Figure 5, top right), and the average length of an inpatient admission decreased from 8.4 days in 1994 to 6.3 days in 2018. The number of ALD-related outpatient visits per person-year was around 1 throughout the study period, with no apparent time trend (Figure 5, bottom left). The number of all-cause emergency room visits for patients with ALD per person-year decreased gradually from 2.59 in 1995 to 0.82 in 2018 (Figure 5, bottom right). In general, the rates of hospital care were higher for men than for women (Figure 5).

In 1994, the route of diagnosis was emergent in $62 \%$ of patients and elective in $38 \%$ of patients. The elective route of diagnosis became the commonest in 2014, and in 2018, $60 \%$ of patients were diagnosed during elective care (Supplementary Figure S3).

\section{Mortality}

The overall mortality after first ALD diagnosis was 27.5\% (95\% confidence interval [CI]: 27.1-27.9\%) after 1 year and 53.9\% (95\% CI: 53.4-54.4\%) after 5 years. For patients diagnosed in 2014-2018 compared to patients diagnosed in 1994-1998, the adjusted RR was 0.83 (95\% CI: $0.78-0.87$ ) for 1-year mortality and 0.93 (95\% CI: $0.90-0.97)$ for 5-year mortality (Table 1). Compared to ALD steatosis, patients with ALD cirrhosis had the highest crude mortality risk and the highest adjusted RRs of mortality of 3.10 (95\% CI: 2.863.37) after 1 year and 2.00 (95\% CI: 1.92-2.09) after 5 years (Supplementary Figure S4 and Table 1), and men had higher mortality than women with adjusted RRs of 1.18 (95\% CI: $1.14-1.23)$ for 1-year mortality and 1.13 (95\% CI: 1.11-1.15) for 5-year mortality. We observed a decrease in 1-, 3-, and 5-year mortality from 2010 to 2015, after adjustment for the effects of sex, age, and ALD diagnosis by standardization (Figure 6). This decrease was less pronounced when stratified by route of diagnosis (Supplementary Figure S5). The 


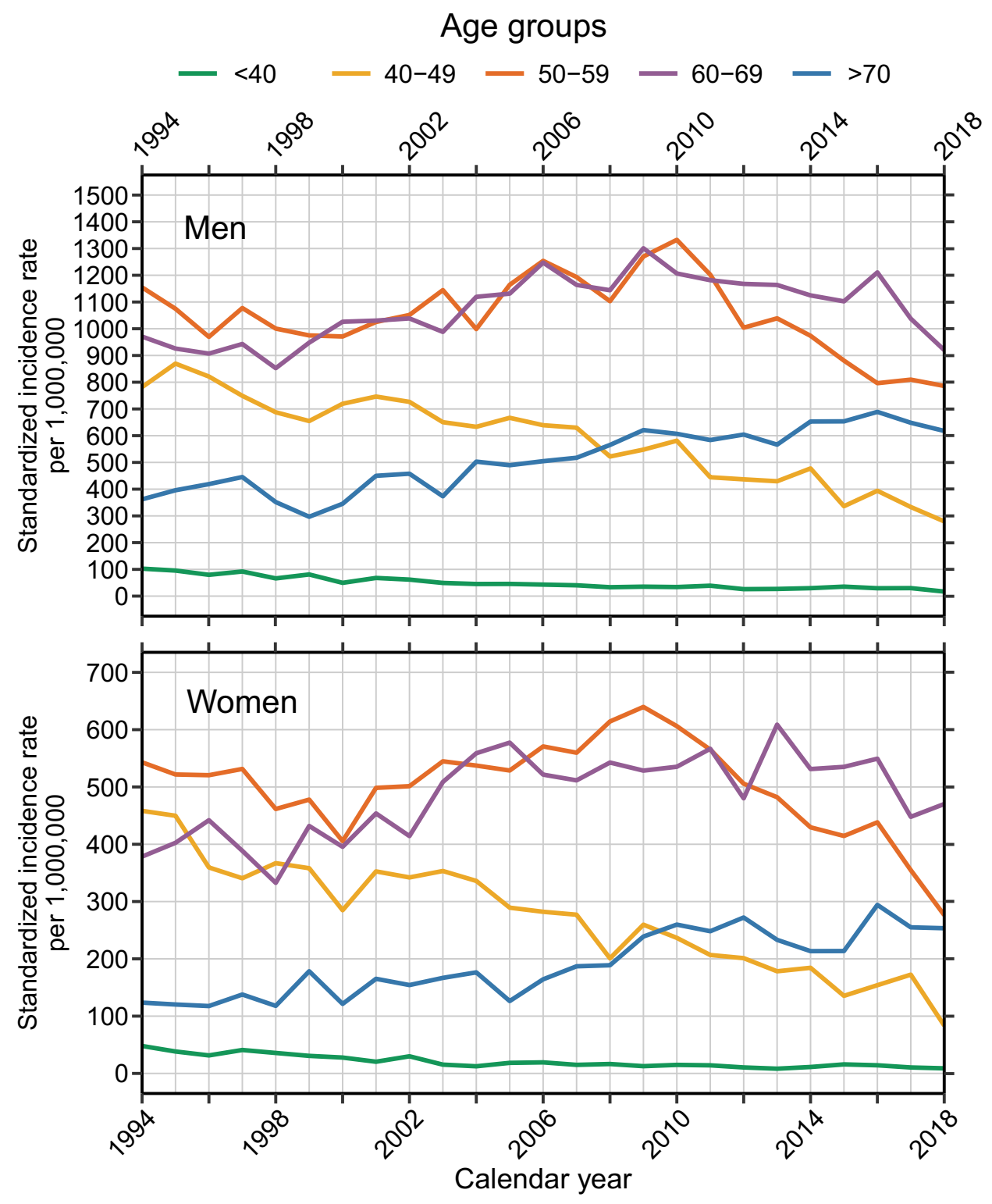

Figure 2 Time trends in annual standardized incidence rates per 1,000,000 Danish population by sex (top/bottom) and age groups (colors).

proportion of 1-year mortality caused by ALD decreased from $75.8 \%$ in 1994 to $60.5 \%$ in 2018 (Figure 6).

\section{Sensitivity Analyses}

By excluding diagnoses of ALD steatosis from the analyses, incidence and prevalence measures decreased by around a tenth, emergency room visits per patient increased by around a tenth, inpatient admissions and outpatient visits per patient increased by around a third, and the proportions of emergent diagnoses and deaths caused by ALD were unchanged. None of the observed time trends were altered.

\section{Alcohol Consumption}

The average annual amount of pure alcohol sold per citizen $>18$ years decreased by $22.4 \%$ from 12.5 liters in 1994 to 9.7 liters in 2018 (Figure 1). The proportion whose alcohol consumption exceeded the high-risk threshold of 252/168 grams of pure alcohol per week (for men/women) decreased from $10.6 \%$ in 2010 to $6.9 \%$ in 2017 (Supplementary Figure S4).

\section{Discussion}

The incidence of ALD decreased since 2009 in Denmark, and the decrease was evident for both sexes and all age 


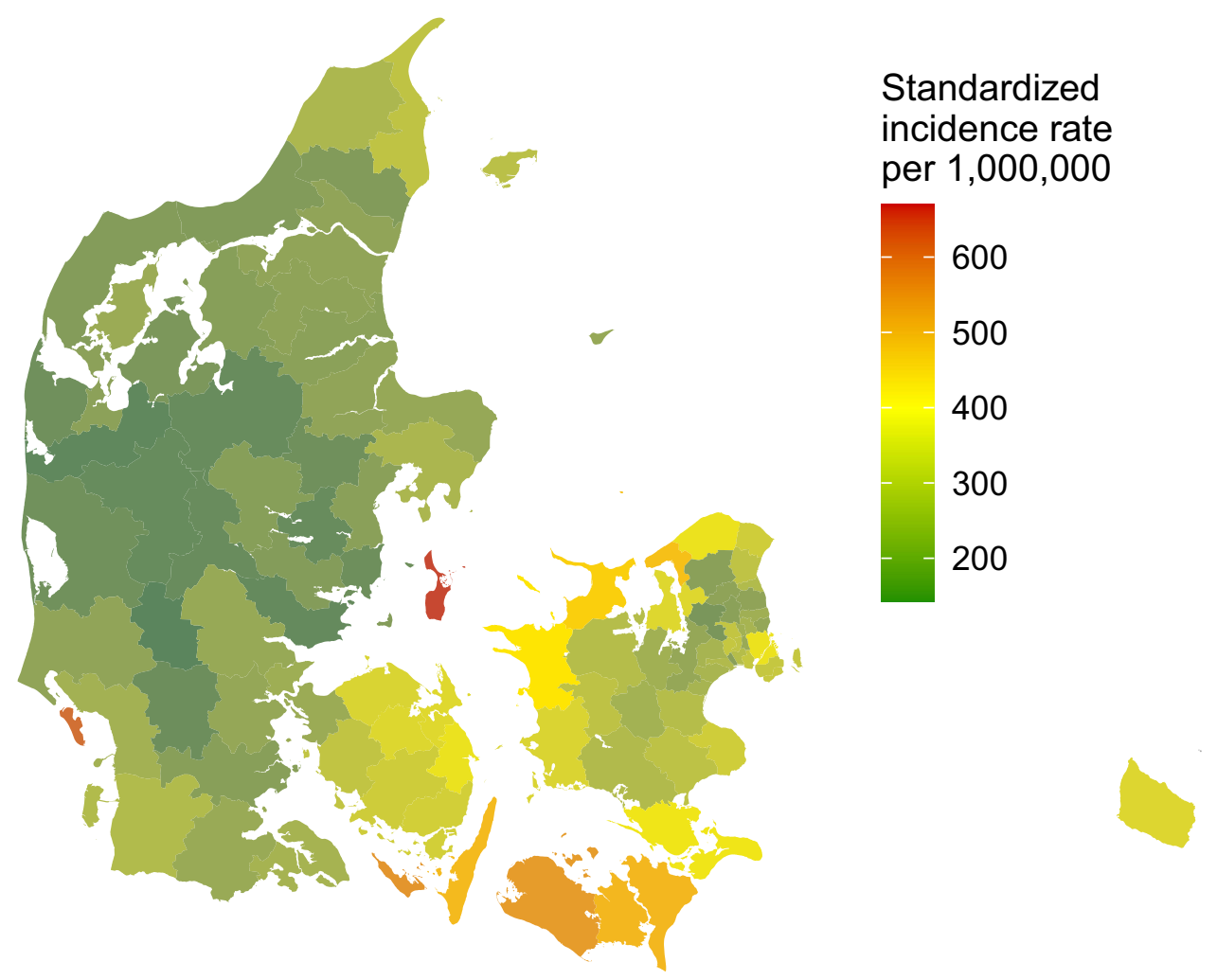

Figure 3 Mean annual incidence rates (2009-2018) by the 98 Danish municipalities standardized to the sex- and age group-distribution of the entire Danish population.

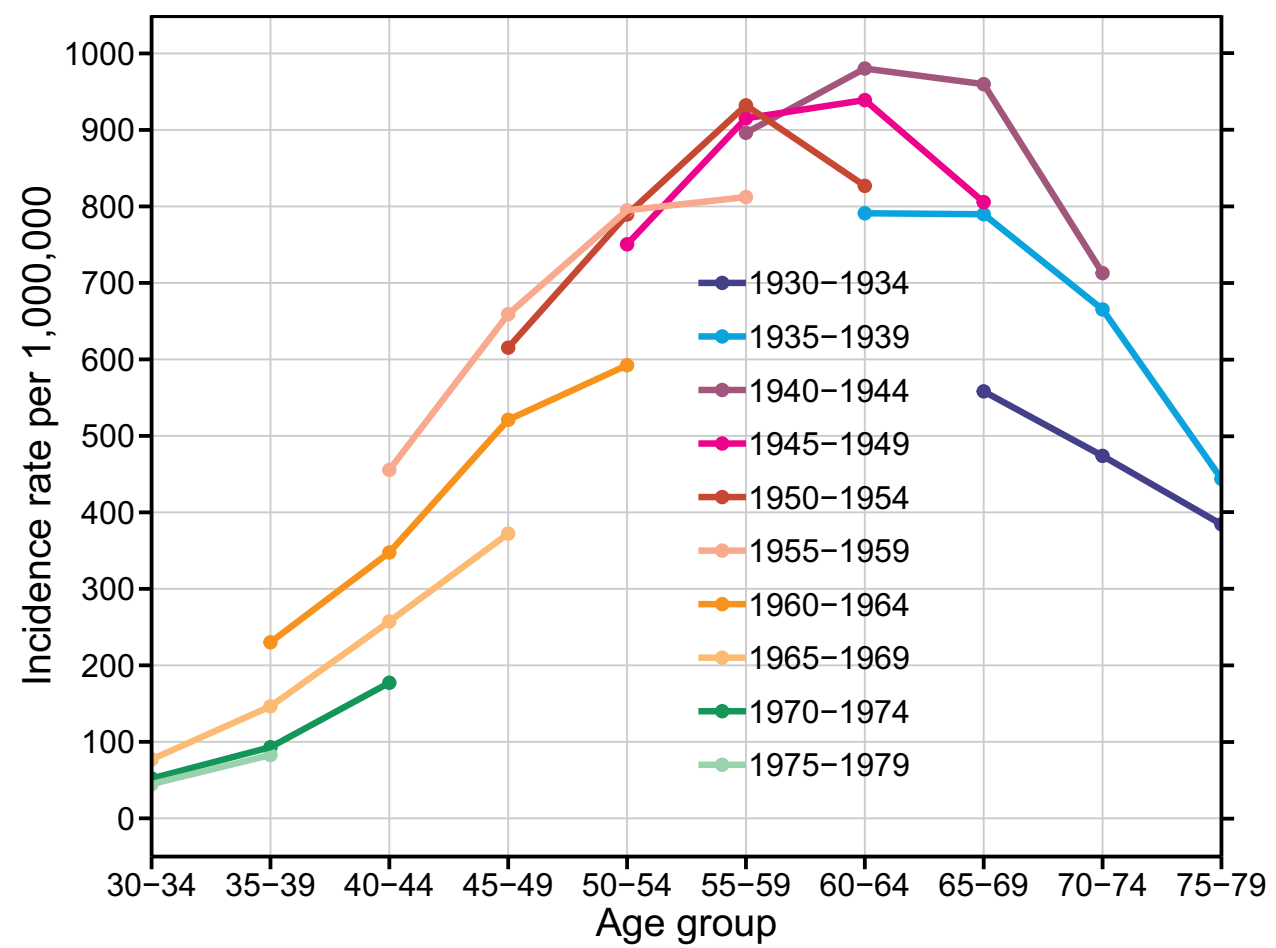

Figure 4 Incidence rates of ALD per 1,000,000 Danish population by 5-year birth cohorts from 1930 to 1979 (colors) and 5-year age groups from 30 to 79 years (x-axis). 

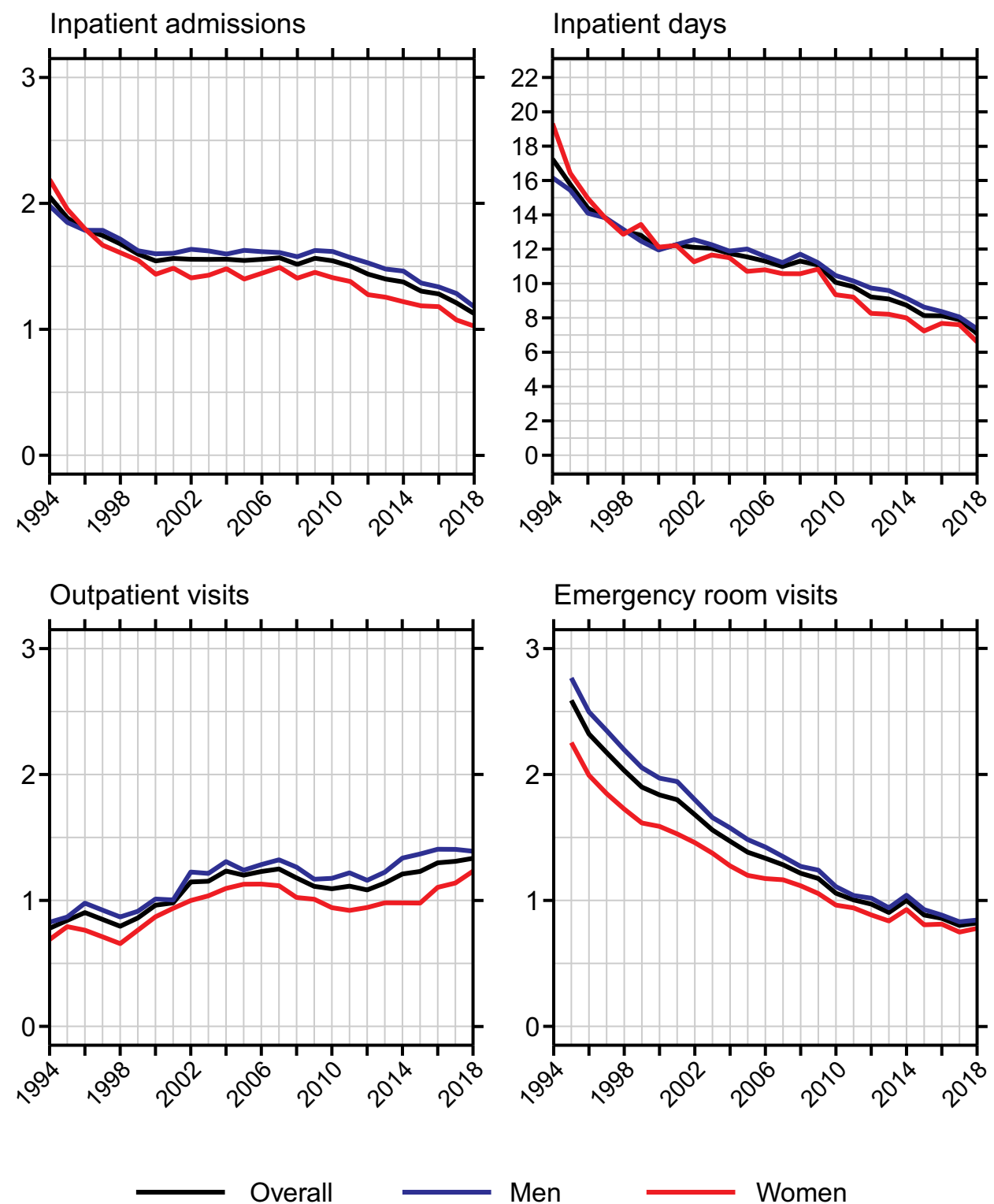

Figure 5 Time trends in hospital care of patients with ALD 1994-2018; the number of all-cause inpatient admissions per patient per person-year (top left). The number of total inpatient days per patient per person-year (top right). The number of ALD-related outpatient visits per patient per person-year (bottom left). The number of all-cause emergency room visits per patient per person-year (bottom right).

groups under 70 years. Within Denmark, there was a nearly fivefold geographic variation in ALD incidence. We saw a decrease in inpatient admissions, emergency room visits, and inpatient days per person-year, while the number of outpatient visits and average length of hospitalization were stable through 2018. Concurrently, the mortality of patients with ALD decreased, which was reflected in a stable prevalence of $0.22 \%$.

It is a strength of this study that we have a single nationwide, public healthcare system with a long history of recording patients' diagnoses. On the other hand, reliance on diagnosis codes is our main concern, since code validity is paramount to the validity of our findings. The codes for liver cirrhosis were validated using medical records in 1997 with a positive predictive value (PPV) of $85.4 \%$ and a completeness of $93.2 \%(n=198) .{ }^{20}$ In 2008 , we found that $78 \%$ of patients with a code for ALD cirrhosis and available liver biopsy data had biopsies containing incipient or manifest cirrhosis, ${ }^{21}$ but in clinical practice, liver biopsies are restricted to patients with uncertain diagnoses; therefore, the true PPV is likely higher. The codes for liver disease in general and had a PPV of $100 \%$ $(n=100)$ in a study from $2011,{ }^{22}$ and the codes for ALD specifically had a PPV of 71\% in another study from 2012 
Table I Mortality After First Alcohol-Related Liver Disease Diagnosis

\begin{tabular}{|c|c|c|c|c|}
\hline & $\begin{array}{l}\text { Crude I-Year } \\
\text { Mortality, \% (95\% } \\
\text { CI) }\end{array}$ & $\begin{array}{l}\text { Crude } 5 \text {-Year } \\
\text { Mortality, \% (95\% } \\
\text { CI) }\end{array}$ & $\begin{array}{l}\text { Adjusted RR for I-Year } \\
\text { Mortality }(95 \% \mathrm{Cl}) \dagger\end{array}$ & $\begin{array}{l}\text { Adjusted RR for } 5 \text {-Year } \\
\text { Mortality }(95 \% \mathrm{Cl}) \dagger\end{array}$ \\
\hline \multicolumn{5}{|l|}{ Sex } \\
\hline Female & $24.3(23.5-25.0)$ & $49.3(48.4-50.2)$ & Ref. & Ref. \\
\hline Male & $29.0(28.5-29.5)$ & $56.0(55.4-56.6)$ & $1.18(1.14-1.23)$ & $1.13(1.11-1.15)$ \\
\hline \multicolumn{5}{|l|}{ Age group } \\
\hline$<40$ years & $14.4(13.0-15.8)$ & $32.2(30.3-34.1)$ & Ref. & Ref. \\
\hline $40-49$ years & $20.7(19.8-21.6)$ & $44.5(43.5-45.6)$ & $1.30(1.18-1-44)$ & $1.28(1.20-1.36)$ \\
\hline $50-59$ years & $24.8(24.1-25.6)$ & $51.7(50.8-52.5)$ & $1.52(1.37-1.68)$ & $1.46(1.38-1.55)$ \\
\hline $60-69$ years & $31.3(30.4-32.1)$ & $59.6(58.7-60.5)$ & $1.93(1.75-2.14)$ & $1.68(1.58-1.79)$ \\
\hline$>70$ years & $44.1(42.8-45.5)$ & $74.8(73.5-76.1)$ & $2.76(2.49-3.05)$ & $2.09(1.96-2.22)$ \\
\hline \multicolumn{5}{|c|}{$\begin{array}{l}\text { Alcohol-related liver } \\
\text { disease diagnosis }\end{array}$} \\
\hline Steatosis & $9.6(8.9-10.4)$ & $28.5(27.3-29.7)$ & Ref. & Ref. \\
\hline Unspecified & $21.5(20.3-22.8)$ & $46.0(44.4-47.6)$ & $2.19(1.98-2.41)$ & $1.57(1.49-1.65)$ \\
\hline Hepatitis & $24.5(23.0-26.1)$ & $45.9(44.1-47.7)$ & $2.60(2.35-2.88)$ & $1.64(1.56-1.74)$ \\
\hline Cirrhosis & $32.4(31.9-33.0)$ & $61.2(60.6-61.8)$ & $3.10(2.86-3.37)$ & $2.00(1.92-2.09)$ \\
\hline \multicolumn{5}{|c|}{ Calendar time period } \\
\hline $1994-1998$ & $25.4(24.5-26.4)$ & $49.9(48.8-51.1)$ & Ref. & Ref. \\
\hline 1999-2003 & $26.9(26.0-27.9)$ & $53.5(52.4-54.6)$ & $1.00(0.95-1.05)$ & $1.03(1.00-1.07)$ \\
\hline $2004-2008$ & $30.0(29.0-31.0)$ & $57.3(56.2-58.3)$ & $1.05(1.00-1.10)$ & $1.07(1.03-1.10)$ \\
\hline $2009-2013$ & $29.0(28.0-29.9)$ & $55.1(54.1-56.2)$ & $0.97(0.93-1.02)$ & $0.99(0.95-1.02)$ \\
\hline $2014-2018$ & $25.8(24.9-26.8)$ & $52.8(51.3-54.2)$ & $0.83(0.78-0.87)$ & $0.93(0.90-0.97)$ \\
\hline
\end{tabular}

Note: $\nmid$ Adjusted for all other variables.

using a larger sample $(\mathrm{n}=1166) .{ }^{23}$ In that study, however, the diagnosis codes had to be verified paraclinically or by cirrhosis complications to be considered valid. ${ }^{23}$ The code

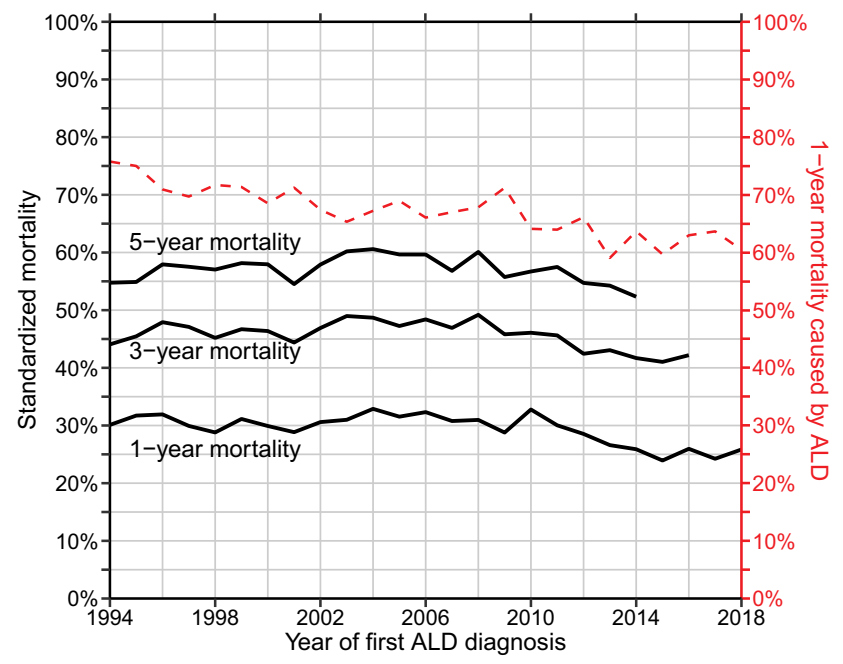

Figure 6 Time trends in sex-, age-, and diagnosis-standardized I-, 3-, and 5-year mortality (left $y$-axis) and the proportion of I-year mortality caused by ALD (right $y$-axis). for alcoholic hepatitis had a PPV of $90 \%$ in a study from $2014(\mathrm{n}=50) .{ }^{24}$ The codes for ALD steatosis have not previously been validated, and the frequency of their use is likely influenced by the degree of clinical ascertainment. Therefore, we conducted sensitivity analyses excluding codes for ALD steatosis. This did not alter any of the observed trends. On top of code validity, we are aware that ALD is under-detected at the hospitals. For instance, $11 \%$ of patients with ALD cirrhosis in Denmark are only diagnosed at death. ${ }^{25}$ However, we find it unlikely that the observed time trends were influenced by either code validity or undetected ALD, since coding practices and the proportion of undetected ALD have remained stable during the ICD-10 era. ${ }^{15}$

In our previous study, we found a somewhat stable ALD incidence, but forecasted a future decrease driven by progressively lower incidence rates in the birth cohorts born after $1960 .{ }^{11}$ In this study, we saw a substantial incidence-decrease during the years following our last study. This finding demonstrates the utility of birth cohort analyses in national forecasts. The age-specific incidence 
is still decreasing sequentially by birth cohorts, and thus, we anticipate a further reduction in the incidence of ALD in Denmark over the coming years. Besides a decreasing incidence, another consequence of higher age-specific incidence rates in older birth cohorts is an aging ALDpopulation, and the median age at diagnosis indeed increased by 10 years during the study period.

Our findings contrast with reports of increasing ALD incidence from Finland, ${ }^{26}$ China $^{27}$ and the United Kingdom. ${ }^{28}$ The authors of these reports point to increased alcohol consumption as a likely cause. Concordantly, our data show that per capita alcohol consumption is correlated with ALD incidence. Hence, the observed decrease in ALD incidence is likely caused by a decreased national alcohol consumption. Regarding other possible causes, there is no evidence suggesting that the decrease in ALD incidence is part of a more general trend in liver disease in Denmark. Actually, the incidence of autoimmune hepatitis has doubled in 1994-2012, ${ }^{29}$ and metabolic associated fatty liver disease has become an increasingly common indication for liver transplantation in the Nordic countries in 1994-2015. ${ }^{30}$ Notably, the decrease in ALD incidence occurred despite the absence of major policy changes or price increases during the last few decades. In fact, Denmark saw a $45 \%$ tax reduction on alcohol in $2003,{ }^{31}$ and since then, the consumer price index of alcoholic beverages has closely followed the overall consumer price index, ${ }^{32}$ meaning that the relative price of alcoholic beverages has remained stable since 2003. The correlation between national policies and alcohol consumption and ALD is currently under scientific scrutiny, but the topic has proved complex. A review of 29 studies of policy changes from five different countries found a general lack of consistent results, ${ }^{9}$ while a comprehensive review commissioned by the UK Department of Health argued that the effect of increased taxation on reducing alcohol consumption is clear. ${ }^{33}$ Our study underscores that alcohol policy is just one of the many factors that may influence national alcohol consumption. In Denmark, in addition to the alcohol tax cut in 2003, we have seen a shift in societal norms with respect to alcohol consumption during the last decades. For instance, it has become more socially unacceptable to drive under the influence of alcohol, ${ }^{34}$ a smaller proportion of road traffic accidents are related to alcohol, ${ }^{21}$ and more women report alcohol abstinence during early pregnancy. ${ }^{35}$ National health campaigns with the aim of reducing alcohol consumption have been run annually by the Danish Health Authority since 1990, but whether these campaigns have influenced societal norms is unclear. $^{36}$

The geographic variation of ALD incidence within Denmark is high. Though social factors may confound the association between ALD incidence and place of residence, the variation may give an indication of specific geographies where better, more targeted public health outreach and education interventions might be required and may inform clinical service planning.

Regarding hospital care, the national numbers from Statistics Denmark (2006-2018) on hospital care for all diseases contrast somewhat with the trends observed in this study. ${ }^{37,38}$ First, the numbers of inpatient admissions and emergency room visits are nationally stable, but have decreased for patients with ALD. Second, outpatient treatment has increased nationally, while outpatient treatment for ALD is stable. Therefore, the trends in hospital care for patients with ALD cannot exclusively be explained by national tendencies.

We found a decrease in all-cause mortality for all ALD patients, as we have previously found for patients with ALD cirrhosis alone after adjusting for several confounders. ${ }^{12}$ Our findings are also compatible with the results from other countries. In the United States, the inhospital mortality rate of ALD has decreased between $2005-2014,{ }^{39}$ and the mortality rate of ALD cirrhosis has decreased between 2004 and 2014. ${ }^{40}$ The cause of the decreased ALD mortality is difficult to ascertain. Nevertheless, our data provide a few hints. First, the route of diagnosis has changed from a predominantly emergent route to a more elective route. Second, the elective route is associated with a significantly lower mortality, which is in line with a prior study on liver cirrhosis survival. ${ }^{41}$ Third, the proportion of 1-year mortality caused by ALD has decreased. This suggests that a shift towards less severe disease at the time of diagnosis has occurred, and the improved overall prognosis is at least in part explained by earlier diagnosis. Despite the improved prognosis, the mortality associated with ALD is still very high, and the fact that over two-thirds of patients have end-stage liver disease (cirrhosis) at the time of diagnosis tells us that there is further potential to reduce the overall mortality by earlier intervention. We have previously found that $40 \%$ of patients who are diagnosed with ALD cirrhosis have at least one prior hospital contact with alcohol problems (alcohol intoxication, harmful alcohol use, or alcohol dependence) within a 10 -year period ${ }^{25}$ and that the risk of ALD cirrhosis increases with the number of these 
contacts. $^{42}$ So naturally, hospital contacts with alcohol problems are a potential point of early intervention.

In conclusion, we found a decreasing incidence, a stable prevalence, and a nearly fivefold geographic variation of ALD in Denmark during the last decade. Concurrently, the burden of ALD on hospital care has decreased and the prognosis of ALD has improved. The decrease in ALD incidence is driven by progressively lower incidence rates in people born after 1960 and likely reflects societal changes rather than government policies. Based on birth cohort trends, we anticipate a further decrease in the incidence of ALD. This is good news for public health.

\section{Ethics and Consent Statement}

According to the Danish Data Protection Act, section 10 (Act No. 502 of 23 May 2018), this study based on data from Danish healthcare registries does not require approval from an ethics committee.

\section{Acknowledgments}

The Danish National Health Survey was funded by The Capital Region, Region Zealand, The South Denmark Region, The Central Denmark Region, The North Denmark Region, The Ministry of Health and the National Institute of Public Health, University of Southern Denmark.

\section{Disclosure}

Peter Jepsen was supported by a grant from the Novo Nordisk Foundation (NNF18OC0054612). The funding organization was not involved in the design and conduct of the study, or in the decision to submit the manuscript for publication. All other authors report no financial support. The authors report no other conflicts of interest in this work.

\section{References}

1. O'Shea RS, Dasarathy S, McCullough AJ. Practice Guideline Committee of the American Association for the Study of Liver D, the Practice Parameters Committee of the American College of G. Alcoholic liver disease. Hepatology. 2010;51(1):307-328. doi:10. 1002/hep. 23258

2. Shield KD, Rylett M, Rehm J. Public Health Successes and Missed Opportunities. Trends in Alcohol Consumption and Attributable Mortality in the WHO European Region, 1990-2014. Copenhagen: World Health Organization. Regional Office for Europe; 2016.

3. Axley PD, Richardson CT, Singal AK. Epidemiology of alcohol consumption and societal burden of alcoholism and alcoholic liver disease. Clin Liver Dis. 2019;23(1):39-50. doi:10.1016/j.cld.2018. 09.011
4. Ann Kristin K. Life expectancy and disease burden in the Nordic countries: results from the Global Burden of Diseases, Injuries, and Risk Factors Study. 2019;2017.

5. Rehm J, Taylor B, Mohapatra S, et al. Alcohol as a risk factor for liver cirrhosis: a systematic review and meta-analysis. Drug Alcohol Rev. 2010;29(4):437-445. doi:10.1111/j.1465-3362.2009.00153.x

6. Askgaard G, Grønbæk M, Kjær MS, Tjønneland A, Tolstrup JS. Alcohol drinking pattern and risk of alcoholic liver cirrhosis: a prospective cohort study. J Hepatol. 2015;62(5):1061-1067. doi:10. 1016/j.jhep.2014.12.005

7. Hart CL, Morrison DS, Batty GD, Mitchell RJ, Davey Smith G. Effect of body mass index and alcohol consumption on liver disease: analysis of data from two prospective cohort studies. BMJ (Clinical Research Ed). 2010;340:c1240. doi:10.1136/bmj.c1240

8. Simpson RF, Hermon C, Liu B, et al. Alcohol drinking patterns and liver cirrhosis risk: analysis of the prospective UK Million Women Study. Lancet Public Health. 2019;4(1):e41-e48. doi:10.1016/S24682667(18)30230-5

9. Nelson JP, McNall AD. What happens to drinking when alcohol policy changes? A review of five natural experiments for alcohol taxes, prices, and availability. Eur $j$ Health Econ. 2017;18 (4):417-434. doi:10.1007/s10198-016-0795-0

10. Heather N. Can screening and brief intervention lead to population-level reductions in alcohol-related harm? Addict Sci Clin Pract. 2012;7:15. doi:10.1186/1940-0640-7-15

11. Deleuran T, Vilstrup H, Becker U, Jepsen P. Epidemiology of alcoholic liver disease in Denmark 2006-2011: a population-based study. Alcohol Alcohol. 2015;50(3):352-357. doi:10.1093/alcalc/agv003

12. Deleuran T, Vilstrup H, Jepsen P. Decreasing mortality among danish alcoholic cirrhosis patients: a nationwide cohort study. $\mathrm{Am}$ J Gastroenterol. 2016;111(6):817-822. doi:10.1038/ajg.2016.107

13. Statistics Denmark - StatBank.dk/alko2 [database online]. Available from: https://statbank.dk/alko2. Accessed May 27, 2020.

14. Schmidt M, Schmidt SAJ, Adelborg K, et al. The Danish health care system and epidemiological research: from health care contacts to database records. Clin Epidemiol. 2019;11:563-591. doi:10.2147/ CLEP.S179083

15. Schmidt M, Schmidt SA, Sandegaard JL, Ehrenstein V, Pedersen L, Sorensen HT. The Danish National Patient Registry: a review of content, data quality, and research potential. Clin Epidemiol. 2015;7:449-490. doi:10.2147/CLEP.S91125

16. Helweg-Larsen K. The Danish register of causes of death. Scand J Public Health. 2011;39(7_suppl):26-29. doi:10.1177/14034948113 99958

17. Lai MHC, Kwok O-M, Hsiao -Y-Y, Cao Q. Finite population correction for two-level hierarchical linear models. Psychol Methods. 2018;23(1):94-112. doi:10.1037/met0000137

18. Andersen PK, Pohar Perme M. Pseudo-observations in survival analysis. Stat Methods Med Res. 2009;19(1):71-99. doi:10.1177/ 0962280209105020

19. The Danish National Health Survey [database online]: Available from: http://www.danskernessundhed.dk/(date. Accessed April 14, 2020.

20. Vestberg K, Thulstrup AM, Sørensen HT, Ottesen P, Sabroe S, Vilstrup H. Data quality of administratively collected hospital discharge data for liver cirrhosis epidemiology. J Med Syst. 1997;21 (1):11-20. doi:10.1023/A:1022835207287

21. Jepsen P, Vilstrup H, Sorensen HT. Alcoholic cirrhosis in Denmark population-based incidence, prevalence, and hospitalization rates between 1988 and 2005: a descriptive cohort study. BMC Gastroenterol. 2008;8:3.

22. Thygesen SK, Christiansen CF, Christensen S, Lash TL, Sørensen HT. The predictive value of ICD-10 diagnostic coding used to assess Charlson comorbidity index conditions in the population-based Danish National Registry of Patients. BMC Med Res Methodol. 2011;11(1):83. doi:10.1186/1471-2288-11-83 
23. Fialla AD, de Muckadell OBS, Touborg Lassen A. Incidence, etiology and mortality of cirrhosis: a population-based cohort study. Scand J Gastroenterol. 2012;47(6):702-709. doi:10.3109/00365521. 2012.661759

24. Orntoft NW, Sandahl TD, Jepsen P, Vilstrup H. Short-term and long-term causes of death in patients with alcoholic hepatitis in Denmark. Clin Gastroenterol Hepatol. 2014;12(10):1739-1744.e17 31. doi:10.1016/j.cgh.2014.04.020

25. Askgaard G, Neermark S, Leon DA, Kjær MS, Tolstrup JS. Hospital contacts with alcohol problems prior to liver cirrhosis or pancreatitis diagnosis. World J Hepatol. 2017;9(36):1332-1339. doi:10.4254/wjh. v9.i36.1332

26. Sahlman P, Nissinen M, Pukkala E, Farkkila M. Incidence, survival and cause-specific mortality in alcoholic liver disease: a population-based cohort study. Scand J Gastroenterol. 2016;51 (8):961-966. doi:10.3109/00365521.2016.1157889

27. Wang W-J, Xiao P, Xu H-Q, Niu J-Q, Gao Y-H. Growing burden of alcoholic liver disease in China: a review. World $J$ Gastroenterol. 2019;25(12):1445-1456. doi:10.3748/wjg.v25.i12.1445

28. Williams R, Aithal G, Alexander GJ, et al. Unacceptable failures: the final report of the Lancet Commission into liver disease in the UK. Lancet (London, England). 2020;395(10219):226-239. doi:10.1016/ S0140-6736(19)32908-3

29. Grønbæk L, Vilstrup H, Jepsen P. Autoimmune hepatitis in Denmark: incidence, prevalence, prognosis, and causes of death. A nationwide registry-based cohort study. J Hepatol. 2014;60(3):612-617. doi:10. 1016/j.jhep.2013.10.020

30. Holmer M, Melum E, Isoniemi $\mathrm{H}$, et al. Nonalcoholic fatty liver disease is an increasing indication for liver transplantation in the Nordic countries. Liver Int. 2018;38(11):2082-2090. doi:10.1111/ liv. 13751

31. Grittner U, Gustafsson N-K, Bloomfield K. Changes in alcohol consumption in denmark after the tax reduction on spirits. Eur Addict Res. 2009;15(4):216-223. doi:10.1159/000239415

32. Statistics Denmark - StatBank.dk/pris111. Available from: https:// statbank.dk/pris111. Accessed November 13, 2020.
33. Burton R, Henn C, Lavoie D, et al. A rapid evidence review of the effectiveness and cost-effectiveness of alcohol control policies: an English perspective. Lancet (British Edition). 2017;389(10078): 1558-1580.

34. Rasmussen SR. Kultur Og Holdninger Til Kørsel I Påvirket Og/Eller Svakket Tilstand [Culture and Opinions on Driving Under the Influence And/Or an Impaired State]. The Danish Center for Social Science Research; 2018.

35. Kesmodel US, Petersen GL, Henriksen TB, Strandberg-Larsen K. Time trends in alcohol intake in early pregnancy and official recommendations in Denmark, 1998-2013. Acta Obstet Gynecol Scand. 2016;95(7):803-810. doi:10.1111/aogs.12890

36. Sundhedsstyrelsen. Er Der Effekt Af Forebyggelseskampagner? [Is There an Effect of Preventive Campaigns?]. Sundhedsstyrelsen; 2013.

37. Statistics Denmark - StatBank. Available from: https://statbank.dk/ indamp02. Accessed May 27, 2020.

38. Statistics Denmark - StatBank. Available from:https://statbank.dk/ skad01. Accessed December 22, 2020.

39. Kim D, Cholankeril G, Li AA, et al. Trends in hospitalizations for chronic liver disease-related liver failure in the United States, 2005-2014. Liver Int. 2019;39(9):1661-1671. doi:10.1111/liv.14135

40. Orman ES, Roberts A, Ghabril M, et al. Trends in characteristics, mortality, and other outcomes of patients with newly diagnosed cirrhosis. JAMA Netw Open. 2019;2(6):e196412. doi:10.1001/ jamanetworkopen.2019.6412

41. Ratib S, Fleming KM, Crooks CJ, Aithal GP, West J. 1 and 5 year survival estimates for people with cirrhosis of the liver in England, 1998-2009: a large population study. J Hepatol. 2013;60(2):282-289. doi:10.1016/j.jhep.2013.09.027

42. Askgaard G, Tolstrup JS, Kjær MS, Leon DA. Number of hospital contacts with alcohol problems predicts later risk of alcoholic liver cirrhosis. Scand J Public Health. 2018;47(4):417-419. doi:10.1177/ 1403494818763436
Clinical Epidemiology

\section{Publish your work in this journal}

Clinical Epidemiology is an international, peer-reviewed, open access, online journal focusing on disease and drug epidemiology, identification of risk factors and screening procedures to develop optimal preventative initiatives and programs. Specific topics include: diagnosis, prognosis, treatment, screening, prevention, risk factor modification,

Submit your manuscript here: https://www.dovepress.com/clinical-epidemiology-journal systematic reviews, risk \& safety of medical interventions, epidemiology \& biostatistical methods, and evaluation of guidelines, translational medicine, health policies \& economic evaluations. The manuscript management system is completely online and includes a very quick and fair peer-review system, which is all easy to use. 